\title{
Multivariate Kurtosis as a Tool for Comparing Copula Models
}

\author{
Ampalavanar Nanthakumar \\ Department of Mathematics, State University of New York at Oswego, Oswego NY 13126, U.S.A. E-mail: \\ ampala.nanthakumar@oswego.edu
}

Received: March 26, 2016 Accepted: April 14, 2016 Online Published: June 22, 2016

doi:10.5539/ijsp.v5n4p67

URL: http://dx.doi.org/10.5539/ijsp.v5n4p67

\begin{abstract}
This paper studies the effectiveness of the Multivariate Kurtosis in comparing the Clayton Copula and the Farleigh-Gumbel-Morgenstern Copula in modeling when the actual populations follow either the bivariate exponential distribution or the bivariate normal distribution. The study shows that the Multivariate Kurtosis (as defined by Mardia) is a very effective tool in comparing Copulas and that Farleigh-Gumbel-Morgenstern Copula is slightly more accurate than the Clayton Copula for modeling.
\end{abstract}

Keywords: Multivariate, Kurtosis, Copula

\section{Introduction}

The copulas are used as a general way of formulating a multivariate distribution in such a way that the dependence can be infused in a reasonable manner. This is based on the simple idea that the joint distribution can be represented as a transformation of the underlying marginal distributions (see Sklar 1959). There are several types of copulas and each differ according to the strength of the dependence and the direction of the association. Ali et al (1978) studied the use of Copulas to construct the bivariate logistic distribution. Aas et al (2009) and Low et al (2013) investigated the applicability of paired (or "Vine") Copulas in the context of finance and portfolio management. Schölzel,and Friederichs (2008) studied the use of Copulas in climatology. Heinen and Rengifo (2007), Nikoloulopoulos and Karlis (2009, 2010), Karlis and Pedeli (2013) constructed bivariate integer-valued autoregressive models based on Copulas. For further literature review, the interested readers are referred to Nelson (2006). Due to the availability of many Copula models and its applications in many subject fields, there is a lot of interest in identifying the "best" Copula model. The question is "How do you compare these different Copula models ?". Here in this paper, we want to compare two particular copulas namely; Clayton Copula, and Farleigh-Gumbel-Morgenstern Copula. The Clayton Copula belongs to the family of Archimedean Copulas while the Farleigh-Gumbel-Morgenstern Copula does belong to the non-Archimedean family. We consider these copulas in the context of modeling the bivariate exponential distribution and the bivariate normal distribution. In order to compare these two Copulas, we use kurtosis as a tool. The kurtosis has been in use for a long time to study the 'peakedness' of the probability distributions. In fact, it is used as a measure to identify the distributions. For example, for the normal distribution the kurtosis is 3 , for the bivariate normal distribution the kurtosis is 8 , for the exponential distribution the kurtosis is 9 , and so on.

In multivariate statistical analysis, normality of the sample is assumed in many cases. Hence, assessing for multivariate normality is an important problem. Similarly, in actuarial models, the bivariate exponential distribution is important and so verification of bivariate exponential is equally important. For this purpose, we will use the bivariate kurtosis as defined in Mardia (1970). In fact, there are several definitions for the Multivariate kurtosis (See, Mardia (1970), Malkovich and Afifi (1973), Srivastava (1984), and Mardia (1970, 1974)). Mardia defined multivariate kurtosis as a natural extension of the univariate case. To assess the multivariate normality, multivariate kurtosis has been defined and its asymptotic distributions under the multivariate normality have been given in Mardia (1970). Furthermore, Srivastava (1984) has considered another definition by using principal component scores and has derived their asymptotic distributions under the null hypothesis. Recently, Miyagawa et al (2011) proposed a sample measure of multivariate kurtosis of the form containing Mardia (1970) and Srivastava (1984). According to Mardia (1970), the multivariate kurtosis can be defined as $\beta_{2}=E\left((X-\mu)^{\prime} \Sigma^{-1}(X-\mu)\right)^{2}$ where $X$ is the observation vector; $\mu$ is the mean vector; 
and $\Sigma$ is the variance-covariance matrix.

In this paper, we propose a method to compare the copulas by using the bivariate kurtosis. We compare the copula through numerical calculations and graphs. Moreover, we check the effectiveness of this approach by estimating the Euclidean distance between the Copula and the actual distribution. The multivariate kurtosis seems to be a good measure for comparing the Copulas.

\section{Methodology}

Copulas:

According to the theory of probability, every joint probability distribution is a function of the marginal distributions (see Sklar (1959) for the details). Copula is a probability model that gives us a way to construct the joint distribution from the marginal distributions. In other words, the Copulas are mathematical models that approximate the multivariate distribution function. There are two families of Copula; Archimedean family of Copulas and the non-Archimedean family of Copulas. Each family consists of several kinds of Copulas. Some of these Copulas are of the discrete type while others are of continuous type.

Next, we present the definitions and the methodology necessary for the construction of the Copulas.

Definition:

A copula is a multivariate joint distribution defined on the $k$ dimensional unit cube $[0,1]^{k}$ such that every marginal distribution is uniform on the interval $[0,1]$.

In other words, $C:[0,1]^{k} \rightarrow[0,1]$ is a $k$-dimensional copula if

(a). $C(u)=0$ whenever $u \in[0,1]^{k}$ has at least one component equal to 0 .

(b). $C(u)=u_{i}$ whenever $u \in[0,1]^{k}$ has all the components equal to 1 except the $i^{t h}$ one which is equal to $u_{i}$.

(c). $C(u)$ is $k$-increasing.

\section{Copula Construction}

Archimedean Copula:

This is a family of copulas and the $k$-dimensional Archimedean Copula is defined as follows.

$$
C\left(u_{1}, u_{2}, \ldots \ldots, u_{k}\right)=\Psi^{-1}\left(\sum_{i=1}^{k} \Psi\left(u_{i}\right)\right)
$$

where $\Psi$ is known as the generator function and $u_{i}$ is the marginal distribution of the $i^{\text {th }}$ component.

Any generator function which satisfies the following properties is the basis for a copula.

$$
\Psi(1)=0, \text { limit } \Psi(x)=\infty, \quad \Psi^{\prime}(x)<0, \Psi^{\prime \prime}(x)>0 \quad x \rightarrow 0
$$

Special Case:

Clayton Copula

Let the generator function $\Psi(u)=u^{-\alpha}-1$ where $\alpha>1$. One can show that the functional inverse,

$$
\Psi^{-1}(u)=(1+u)^{-1 / \alpha}
$$

In the bivariate case, the Archimedean formulation yields the Clayton Copula as

$$
C\left(u_{1}, u_{2}\right)=\left(u_{1}^{-\alpha}+u_{2}^{-\alpha}-1\right)^{-1 / \alpha}
$$




\section{Non-Archimedian Copula:}

The non-Archimedian copulas do not possess a generator function. These copulas are distinctly different from the Archimedian copulas.

Special Case:

Farleigh-Gumbel-Morgenstern Copula:

$$
C\left(u_{1}, u_{2}\right)=u_{1} \cdot u_{2} \cdot\left(1+\alpha \cdot\left(1-u_{1}\right) \cdot\left(1-u_{2}\right)\right)
$$

where $\alpha$ is the dependence parameter and $|\alpha| \leq 1$.

Modeling the Joint Distribution by Copulas:

Let us suppose that the joint distribution of $(X, Y)$ is $F(x, y)$ is unknown, but the marginal distributions are known.

Note that $F(x, y)$ is the joint distribution.

Let $u_{1}=F_{1}(X)$ and $u_{2}=F_{2}(Y)$ where $F_{1}$ and $F_{2}$ are the marginal distributions of $X$ and $Y$ respectively.

Theorem \#1 (Sklar):

If $X$ and $Y$ are continuous random variables then there exists a continuous Copula $C\left(u_{1}, u_{2}\right)$ such that

$$
C\left(u_{1}, u_{2}\right)=F(x, y)
$$

Theorem \#2:

The conditional distribution of $Y$ given $X=\frac{\partial C\left(u_{1}, u_{2}\right)}{\partial u_{1}}$

Note: We will use the above results in Copula modeling.

Our objective here is to see whether the Copula models nearly resemble the actual populations and to identify the populations as either as "normal" or "non-normal" based on the kurtosis. Towards this, we will consider the bivariate exponential and the bivariate normal populations in this paper.

First, we will consider the bivariate exponential population. In other words, the bivariate observations $\left(x_{i}, y_{i}\right)$ follows the bivariate exponential distribution with the cumulative distribution function (cdf) given by

$$
F(x, y)=1-e^{-\lambda_{1} \cdot x}-e^{-\lambda_{2} \cdot y}-e^{-\lambda_{1} \cdot x-\lambda_{2} \cdot y+\lambda \cdot \min (x, y)}
$$

In the absence of any knowledge about the actual population distribution, we propose to use the Copulas to model this population distribution based on the marginal distributions. Here, we assume that the marginal distributions are known.

Say, that for the $X$ variable, its marginal distribution $F_{1}(x)$ is given by $F_{1}(x)=1-e^{-\lambda_{1} \cdot x}$. Similarly, the marginal distribution of the $Y$ variable is given by $F_{2}(y)=1-e^{-\lambda_{\cdot 2} y}$.

Modeling Clayton Copula:

$$
C\left(u_{1}, u_{2}\right)=\left(u_{1}^{-\alpha}+u_{2}^{-\alpha}-1\right)^{\frac{-1}{\alpha}}
$$


where $u_{1}$ and $u_{2}$ are the marginal distributions of $X$ and $Y$ respectively.

In order to generate the Clayton Copula, we will use Theorem \#2.

For the Clayton Copula,

$$
\frac{\partial C}{\partial u_{1}}=\left(u_{1}^{-\alpha}+u_{2}^{-\alpha}-1\right)^{\frac{-1}{\alpha}} \cdot u_{1}^{-\alpha}=v \quad \text { (say) }
$$

where $v$ is the conditional distribution of $Y$ given $X$. So, $v$ is uniformly distributed between 0 and 1 .

This means that,

$$
u_{2}=F_{2}(y)=\left\{1-u_{1}^{-\alpha} \cdot\left(1+v^{\frac{-\alpha}{(\alpha+1)}}\right)\right\}^{\frac{-1}{\alpha}}
$$

So, $1-e^{-\lambda_{2} \cdot y}=\left\{1-u_{1}^{-\alpha}\left(1+v^{\frac{-\alpha}{(\alpha+1)}}\right)\right\}^{\frac{-1}{\alpha}}$

and $y=\frac{-1}{\lambda_{2}} \cdot \ln \left\{1-\left(1-u_{1}^{-\alpha}\left(1+v^{\frac{-\alpha}{(\alpha+1)}}\right)\right)^{\frac{-1}{\alpha}}\right\}$

Also, note that $u_{1}=1-e^{-\lambda_{1} x}$

This means that,

$$
x=\frac{-1}{\lambda_{1}} \cdot \ln \left\{1-u_{1}\right\}
$$

Note that $\alpha$ is the dependence parameter and it can be estimated by using the relationship that Kendall's Tau, $\tau=\frac{\alpha}{(\alpha+2)}$. By using equations (2.10) and (2.11) the Clayton copula based samples can be generated.

Modeling Farleigh-Gumbel-Morgenstern Copula:

$$
C\left(u_{1}, u_{2}\right)=u_{1} \cdot u_{2} \cdot\left(1+\alpha \cdot\left(1-u_{1}\right) \cdot\left(1-u_{2}\right)\right)
$$

where $\alpha$ is the dependence parameter and $|\alpha| \leq 1$.

Note that $\frac{\partial C}{\partial u_{2}}=u_{1}+\alpha \cdot\left(u_{1}-u_{1}^{2}\right) \cdot\left(1-2 \cdot u_{2}\right)=z$

where $z$ represents the conditional distribution of $X$ given $Y=y$.

This yields the quadratic equation,

$$
\alpha .\left(1-2 \cdot u_{2}\right) \cdot u_{1}^{2}-\left\{1+\alpha \cdot\left(1-2 \cdot u_{2}\right)\right\} \cdot u_{1}+z=0
$$


The solution is given by

$$
u_{1}=\frac{\left\{1+\alpha \cdot\left(1-2 \cdot u_{2}\right)\right\}-\sqrt{\left\{1+\alpha \cdot\left(1-2 \cdot u_{2}\right)\right\}^{2}-4 \cdot \alpha \cdot\left(1-2 \cdot u_{2}\right) \cdot z}}{2 \cdot \alpha \cdot\left(1-2 \cdot u_{2}\right)}
$$

Note that, $u_{1}=1-e^{-\lambda_{1} \cdot x}$ and $u_{2}=1-e^{-\lambda_{2} \cdot y}$

Now, let $A=1+\alpha \cdot\left(1-2 \cdot u_{2}\right)$ and $B=\left[1+\alpha \cdot\left(1-2 \cdot u_{2}\right)\right]^{2}-4 \cdot \alpha \cdot\left(1-2 \cdot u_{2}\right) \cdot z$

This means,

$x=\frac{1}{\lambda_{1}} \cdot \ln \left\{\frac{2 \cdot(A-1)}{A+\sqrt{B}-2}\right\}$ and $y=\frac{-\ln \left(1-u_{2}\right)}{\lambda_{2}}$

Note that again $\alpha$ is the dependence parameter and it can be estimated by using the relationship that the Pearson Coefficient of Correlation $\rho=\frac{\alpha}{4}$. By using equation (2.17), Farleigh-Gumbel-Morgenstern copula based samples can be generated.

Next, in order to simulate the samples directly from the Bivariate Exponential distribution, we will do the following.

Let us choose a value $\lambda$ such that $\lambda<\min \left\{\lambda_{1}, \lambda_{2}\right\}$. Next, simulate random observations $\tilde{U}$ according to the exponential distribution with mean $=\frac{1}{\left(\lambda_{1}-\lambda\right)}$. Similarly, simulate random observations $\tilde{V}$ according to the exponential distribution with mean $=\frac{1}{\left(\lambda_{2}-\lambda\right)}$. Also, we can simulate $\tilde{W}$ according to an exponential distribution with mean $=\frac{1}{\lambda}$

Now, let us define, $\tilde{X}=\min \{\tilde{U}, \tilde{W}\}$ and $\tilde{Y}=\min \{\tilde{V}, \tilde{W}\}$. We can easily show that $(\tilde{X}, \tilde{Y})$ jointly follow the bivariate exponential distribution as given by $(2.6)$. We are interested in studying two things in this paper.

(i).The suitability of the Copula models to study the covariance structure.

(ii).The use of kurtosis as a tool to check the validity of the Copula models.

Kurtosis:

Let us first introduce the notations that we will use in the context of kurtosis calculation.

$\mu_{X}=$ Mean of $X$

$\mu_{Y}=$ Mean of $Y$

$\Sigma=$ Covariance Matrix of $(X, Y)$

Next, we present Mardia's definition for Kurtosis for the multivariate situation. 
Kurtosis (Mardia)

The bivariate kurtosis, $\kappa$ is given by

$$
\kappa=E\left\{\left(X-\mu_{X}, Y-\mu_{Y}\right) \Sigma^{-1}\left(X-\mu_{X}, Y-\mu_{Y}\right)^{T}\right\}^{2}
$$

Next, to derive the kurtosis, let us note that the inverse of the variance-covariance matrix is

$$
\Sigma^{-1}=\left(\begin{array}{ll}
a_{11} & a_{12} \\
a_{21} & a_{22}
\end{array}\right)
$$

Then, one can easily show that

$$
\begin{gathered}
\kappa=a_{11}{ }^{2} \cdot E\left(X-\mu_{X}\right)^{4}+a_{22}{ }^{2} \cdot E\left(Y-\mu_{Y}\right)^{4}+4 \cdot a_{12}{ }^{2} \cdot E\left(\left(X-\mu_{X}\right)^{2} \cdot\left(Y-\mu_{Y}\right)^{2}\right) \\
+2 \cdot a_{11} \cdot a_{22} \cdot E\left(\left(X-\mu_{X}\right)^{2} \cdot\left(Y-\mu_{Y}\right)^{2}\right)+4 \cdot a_{11} \cdot a_{12} \cdot E\left(\left(X-\mu_{X}\right)^{3} \cdot\left(Y-\mu_{Y}\right)\right) \\
+4 \cdot a_{22} \cdot a_{12} \cdot E\left(\left(X-\mu_{X}\right) \cdot\left(Y-\mu_{Y}\right)^{3}\right)
\end{gathered}
$$

Note that $a_{11}=\sigma_{X}{ }^{-2} \cdot\left(1-\rho^{2}\right)^{-1}$

$$
\begin{aligned}
& a_{22}=\sigma_{Y}^{-2} \cdot\left(1-\rho^{2}\right)^{-1} \\
& a_{12}=\frac{-\rho \cdot\left(1-\rho^{2}\right)^{-1}}{\sigma_{X} \cdot \sigma_{Y}}
\end{aligned}
$$

where $\rho$ is the correlation coefficient between $X$ and $Y$.

Next, we present the following results in order to evaluate the kurtosis. The proofs can be found in the Appendix. If the joint probability distribution is a bivariate normal then

Result 1: $E\left(\left(X-\mu_{X}\right)^{2}\left(Y-\mu_{Y}\right)^{2}\right)=\sigma_{X}{ }^{2} \cdot \sigma_{Y}{ }^{2} \cdot\left(1-\rho^{2}\right)+3 \cdot \rho^{2} \cdot \sigma_{X}{ }^{2} \cdot \sigma_{Y}{ }^{2}$

Result 2: $E\left(\left(X-\mu_{X}\right)^{3}\left(Y-\mu_{Y}\right)\right)=3 \cdot \rho \cdot \sigma_{X}{ }^{3} \cdot \sigma_{Y}$

Result 3: $E\left(\left(X-\mu_{X}\right)\left(Y-\mu_{Y}\right)^{3}\right)=3 \cdot \rho \cdot \sigma_{X} \cdot \sigma_{Y}{ }^{3}$

Result 4: Kurtosis, $\kappa=8$.

If the joint distribution is bivariate exponential with the density function

$$
f(x, y)=\left\{\begin{array}{c}
\lambda_{1} \cdot\left(\lambda_{2}-\lambda\right) \cdot e^{-\lambda_{1} \cdot x-\left(\lambda_{2}-\lambda\right) \cdot y}, x>y \\
\lambda_{2} \cdot\left(\lambda_{1}-\lambda\right) \cdot e^{-\lambda_{2} \cdot y-\left(\lambda_{1}-\lambda\right) \cdot x}, x<y \\
\lambda \cdot e^{-\left(\lambda_{1}+\lambda_{2}-\lambda\right) \cdot y}, x=y
\end{array}\right.
$$

then the following results are true.

Result 5: $E\left(\left(X-\mu_{X}\right)\left(Y-\mu_{Y}\right)\right)=\frac{\lambda}{\lambda_{1} \cdot \lambda_{2} \cdot\left(\lambda_{1}+\lambda_{2}-\lambda\right)}$ 
Result 6: $E\left(\left(X-\mu_{X}\right)^{2} \cdot\left(Y-\mu_{Y}\right)^{2}\right)=\frac{1}{\lambda_{1}{ }^{2} \cdot \lambda_{2}{ }^{2}}+\frac{8 \cdot \lambda}{\lambda_{1} \cdot \lambda_{2} \cdot\left(\lambda_{1}+\lambda_{2}-\lambda\right)^{3}}$

Result 7: $E\left(\left(X-\mu_{X}\right)^{3} \cdot\left(Y-\mu_{Y}\right)\right)=\frac{3 \cdot \lambda}{\lambda_{1}^{3} \cdot \lambda_{2} \cdot\left(\lambda_{1}+\lambda_{2}-\lambda\right)}+\frac{6 \cdot \lambda}{\lambda_{1} \cdot \lambda_{2} \cdot\left(\lambda_{1}+\lambda_{2}-\lambda\right)^{3}}$

Result 8: $E\left(\left(X-\mu_{X}\right) \cdot\left(Y-\mu_{Y}\right)^{3}\right)=\frac{3 \cdot \lambda}{\lambda_{1} \cdot \lambda_{2}^{3} \cdot\left(\lambda_{1}+\lambda_{2}-\lambda\right)}+\frac{6 \cdot \lambda}{\lambda_{1} \cdot \lambda_{2} \cdot\left(\lambda_{1}+\lambda_{2}-\lambda\right)^{3}}$

Result 9: Kurtosis, $\kappa=20+\frac{20 \cdot \rho^{2}}{\left(1-\rho^{2}\right)}+\frac{8 \cdot \lambda \cdot\left\{2 \cdot \lambda_{1} \cdot \lambda_{2} \cdot\left(1+2 \cdot \rho^{2}\right)-3 \cdot \rho \cdot\left(\lambda_{1}^{2}+\lambda_{2}^{2}\right)\right\}}{\left(1-\rho^{2}\right)^{2} \cdot\left(\lambda_{1}+\lambda_{2}-\lambda\right)^{3}}$

Note that the kurtosis will be 20 when the components are independent.

\section{Results}

- Numerical Result:

Kurtosis Calculation (Bivariate Exponential):

The Kurtosis given by $(2.20)$ is estimated for the data generated from the Morgenstern Copula and the Clayton Copula and in addition to the simulated data from the bivariate exponential distribution by using the following parameters.

$$
\lambda_{1}=0.4, \quad \lambda_{2}=0.3 \quad, \quad \lambda=0.1 \quad, \alpha=0.7
$$

Kurtosis Estimate $($ simulated bivariate exponential $)=18.036$

Kurtosis $($ Actual $)=21.086$

Kurtosis Estimate (Morgenstern Copula) $=22.585$

Average distance estimate (of Morgenstern Copula from actual Population) $=5.631$

Kurtosis Estimate (Clayton Copula) $=30.036$

Average distance estimate (of Clayton Copula from actual Population) $=5.927$

\section{- Graphical Result (Bivariate Exponential):}

Here, we present the scatterplots based on the copulas. The blue dots in the scatterplot represent the data that was simulated from the bivariate exponential distribution with the sample size $=1000$. The red dots in the scatterplot represent the data that was generated by using the Morgenstern Copula while the green dots represent the data generated from the Clayton Copula.

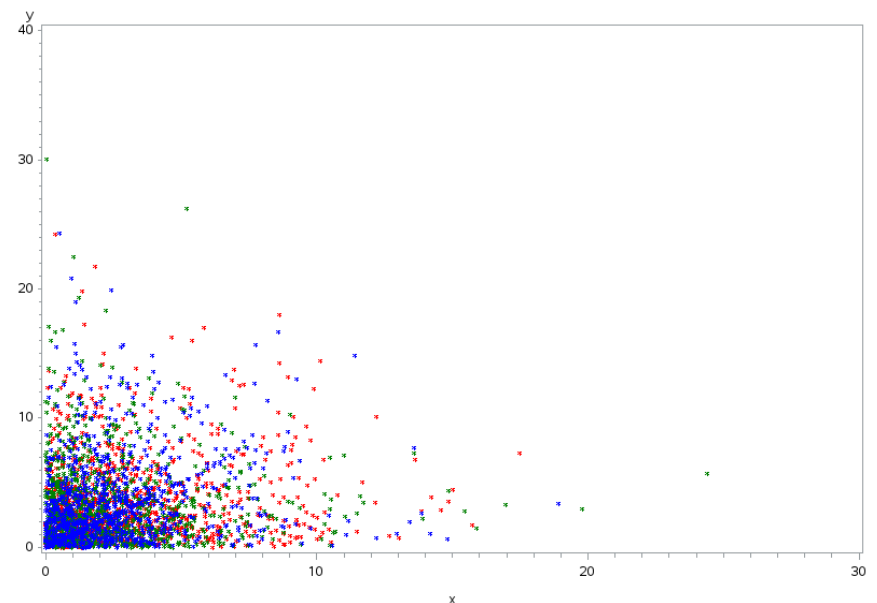


- $\quad$ Kurtosis Calculation (Bivariate Normal):

The Kurtosis given by $(2.20)$ is estimated for the data generated from the Morgenstern Copula and the Clayton Copula and in addition to the simulated data from the bivariate normal distribution by using the following parameters.

$$
\mu_{1}=2.25, \mu_{2}=3.33, \quad \rho=0.175, \sigma_{1}=2.25, \sigma_{2}=3.33
$$

Kurtosis Estimate (simulated bivariate normal) $=7.761$

Kurtosis (Actual) $=8$

Kurtosis Estimate (Morgenstern Copula) $=7.735$

Average distance estimate (of Morgenstern Copula from actual Population) $=0.515$

Kurtosis Estimate (Clayton Copula) $=10.165$

Average distance estimate (of Clayton Copula from actual Population) $=5.637$

- Graphical Result (Bivariate Normal):

Here, we again present the scatterplots based on the copulas. The blue dots in the scatterplot represent the data that was simulated from the bivariate normal distribution with the sample size $=1000$. The red dots in the scatterplot represent the data that was generated by using the Morgenstern Copula while the green dots represent the data generated from the Clayton Copula.

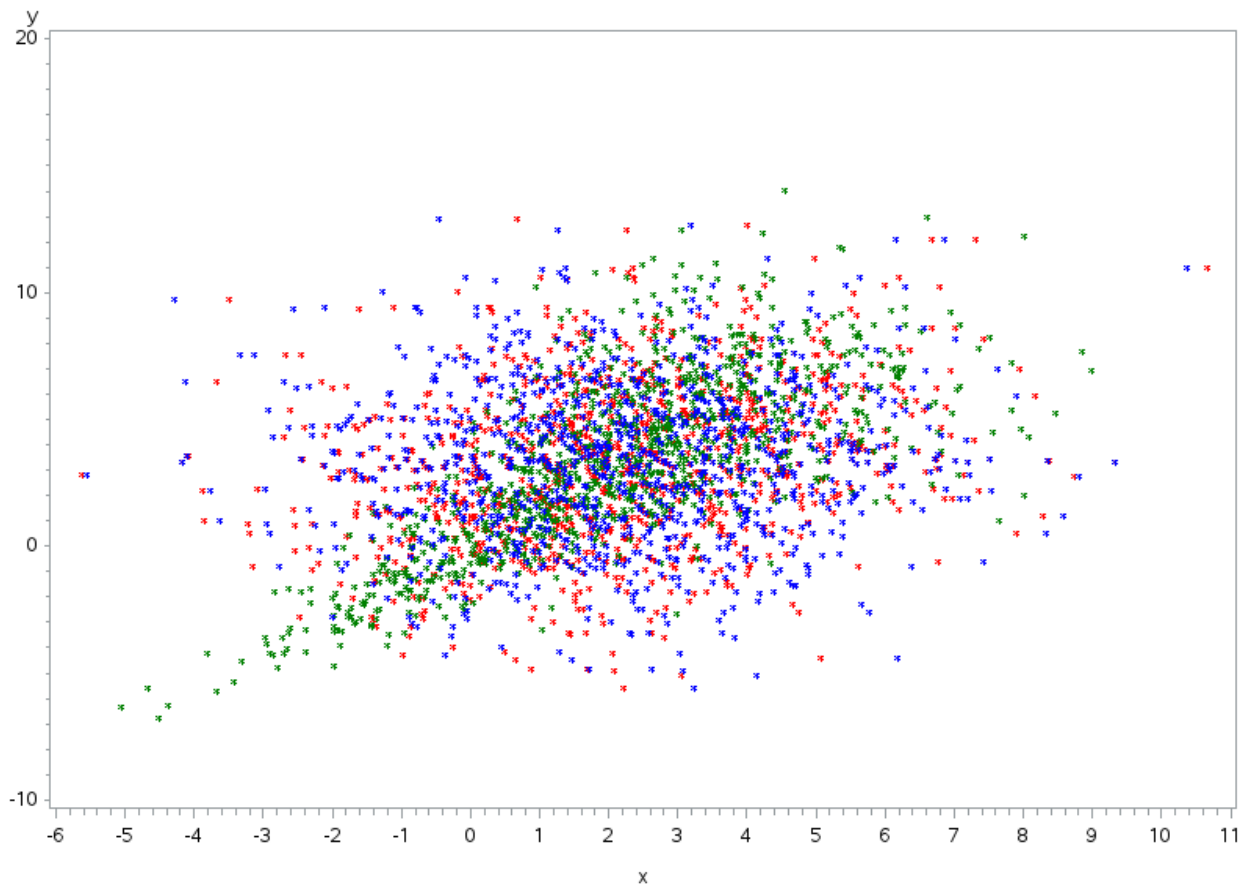

\section{Discussion and Conclusion}

The Copulas allow us to model the multivariate distributions from the marginal distributions. There are many types of Copulas. The Farleigh-Gumbel-Morgenstern Copula is used in Actuarial models and also in Engineering related reliability studies. The Clayton Copula is used mostly in Finance and Marketing. This paper is focused on comparing the Farleigh-Gumbel-Morgenstern Copula with the Clayton Copula. Note that these two copulas belong to two different families with the Clayton Copula coming from the Archiemedian Family and the Farleigh-Gumbel-Morgenstern Copula from the Non-Archemedian Family. We chose the parameters so that the mean vector and the variance-covariance matrix would be the same for the bivariate exponential and the bivariate normal distributions. Note that from the numerical results, it is obvious that the Farleigh-Gumbel-Morgenstern Copula (or simply Morgenstern Copula) is slightly more accurate than the Clayton Copula for modeling both the bivariate exponential distribution and the 
bivariate normal distribution. Also, the scatterplots seemed to support the numerical results based on the Kurtosis. Furthermore, from this paper it is evident that the multivariate kurtosis is a reasonable measure to compare these two Copulas.

\section{References}

Aas, K., Czado, C., \& Bakken, H. (2009). Pair-copula constructions of multiple dependence, Insurance. Mathematics and Economics, 44(2), 182-198.

Ali, M. M., Mikhail, N. N., \& Haq, M. S. (1978). A class of bivariate distributions including the bivariate logistic. J. Multivariate Anal., 8, 405-412.

Heinen, A., \& Rengifo, E. (2007). Multivariate autoregressive modeling of time series count data using copulas. Journal of Empirical Finance, 14, 564 - 583.

Karlis, D., \& Pedeli, X. (2013). Flexible bivariate INAR(1) processes using copulas. Communications in StatisticsTheory and Methods, 42, 723-740.

Low, R., Alcock, J., Brailsford, T., \& Faff, R. (2013). Canonical vine copulas in the context of modern portfolio management: Are they worth it?. Journal of Banking and Finance, 37(8), 3085-3099.

Malkovich, J. F., \& Afifi, A. A. (1973). On tests for multivariate normality. Journal of the American Statistical Association, 68, 176-179.

Mardia, K. V. (1970). Measures of multivariate skewness and kurtosis with applications. Biometrika, 57, 519-530.

Mardia, K. V. (1974). Applications of some measures of multivariate skewness and kurtosis in testing normality and robustness studies. Sankhya B, 36, 115-128.

Miyagawa, C., Koizumi, K., \& Seo, T. (2011). A new multivariate kurtosis and its asymptotic distribution. SUT Journal of Mathematics, 47, 55-71.

Nelson, R. B. (2006). An Introduction to Copulas. Springer.

Nikoloulopoulos, A., \& Karlis, D. (2009). Modeling multivariate count data using copulas. Communications in Statistics- Simulation and Computation, 39(1), 172-187.

Nikoloulopoulos, A., \& Karlis, D. (2010). Regression in a copula model for bivariate count data. Journal of Applied Statistics, 37, 1555-1568

Schölzel, C., \& Friederichs, P. (2008). Multivariate non-normally distributed random variables in climate research introduction to the copula approach. Nonlinear Processes in Geophysics, 15(5), 761-772.

Sklar, A. (1959). Fonctions de repartition a n dimensions et leurs marges. Publications de L'Institut de Statistique de L' Universite de Paris, 8, 229-231.

Srivastava, M. S. (1984). A measure of skewness and kurtosis and a graphical method for assessing multivariate normality. Statistics \& Probability Letters, 2, 263-267.

\section{Appendix:}

Result 1: $\quad E\left(\left(X-\mu_{X}\right)^{2}\left(Y-\mu_{Y}\right)^{2}\right)={\sigma_{X}}^{2} \cdot \sigma_{Y}{ }^{2} \cdot\left(1-\rho^{2}\right)+3 \cdot \rho^{2} \cdot \sigma_{X}{ }^{2} \cdot \sigma_{Y}{ }^{2}$

Proof: Note that, $E\left(\left(X-\mu_{X}\right)^{2} \backslash Y\right)=\sigma_{X}{ }^{2} \cdot\left(1-\rho^{2}\right)+\rho \cdot \mu_{X} \cdot \frac{\sigma_{X}}{\sigma_{Y}} \cdot\left(Y-\mu_{Y}\right)+\rho^{2} \cdot \frac{\sigma_{X}{ }^{2}}{\sigma_{Y}{ }^{2}} \cdot\left(Y-\mu_{Y}\right)^{2}$

This implies that

$$
\begin{gathered}
E\left(\left(X-\mu_{X}\right)^{2} \cdot\left(Y-\mu_{Y}\right)^{2}\right)=E\left\{\left(Y-\mu_{Y}\right)^{2} \cdot E\left(\left(X-\mu_{X}\right)^{2} \backslash Y\right)\right\} \\
=E\left(\sigma_{X}{ }^{2} \cdot\left(1-\rho^{2}\right) \cdot\left(Y-\mu_{Y}\right)^{2}\right)+E\left(\rho \cdot \mu_{X} \cdot \frac{\sigma_{X}}{\sigma_{Y}} \cdot\left(Y-\mu_{Y}\right)^{3}\right)+E\left(\rho^{2} \cdot \frac{\sigma_{X}{ }^{2}}{\sigma_{Y}{ }^{2}} \cdot\left(Y-\mu_{Y}\right)^{4}\right) \\
=\sigma_{X}{ }^{2} \cdot \sigma_{Y}{ }^{2} \cdot\left(1-\rho^{2}\right)+3 \cdot \rho^{2} \cdot \sigma_{X}{ }^{2} \cdot \sigma_{Y}{ }^{2}
\end{gathered}
$$


Result 2: $E\left(\left(X-\mu_{X}\right) \cdot\left(Y-\mu_{Y}\right)^{3}\right)=3 \cdot \rho \cdot \sigma_{X} \cdot \sigma_{Y}{ }^{3}$

Proof: Note that, $E(X \backslash Y)=\mu_{X}+\rho \cdot \frac{\sigma_{X}}{\sigma_{Y}} .\left(Y-\mu_{Y}\right)$

This implies that

$$
\begin{aligned}
E\left(\left(X-\mu_{X}\right) \cdot\left(Y-\mu_{Y}\right)^{3}\right)=E\left\{\left(Y-\mu_{Y}\right)^{3} \cdot E\left(\left(X-\mu_{X}\right) \backslash Y\right)\right\} \\
=E\left\{\left(Y-\mu_{Y}\right)^{3} \cdot \rho \cdot \frac{\sigma_{X}}{\sigma_{Y}} \cdot\left(Y-\mu_{Y}\right)\right\} \\
=\rho \cdot \frac{\sigma_{X}}{\sigma_{Y}} \cdot E\left(Y-\mu_{Y}\right)^{4} \\
=3 \rho \cdot \sigma_{X} \cdot \sigma_{Y}{ }^{3}
\end{aligned}
$$

Result 3: $E\left(\left(X-\mu_{X}\right)^{3} \cdot\left(Y-\mu_{Y}\right)\right)=3 \cdot \rho \cdot \sigma_{X}{ }^{3} \cdot \sigma_{Y}$

Proof: Follows from symmetry.

Result 4: Kurtosis, $\kappa=8$.

Proof: Note that R.H.S of (2.15) can be written as

$$
\begin{gathered}
\left(1-\rho^{2}\right)^{-2} \cdot 3 \frac{\sigma_{X}{ }^{4}}{\sigma_{X}{ }^{4}}+\left(1-\rho^{2}\right)^{-2} \cdot 3 \frac{\sigma_{Y}{ }^{4}}{\sigma_{Y}{ }^{4}}+4 \frac{\rho^{2}}{\sigma_{X}{ }^{2} \cdot \sigma_{Y}{ }^{2}} \cdot \sigma_{X}{ }^{2} \cdot \sigma_{Y}{ }^{2} \cdot\left(1-\rho^{2}\right)^{-1} \\
+4 .\left(\frac{1}{\sigma_{X}{ }^{2}}\right) \cdot\left(\frac{-\rho}{\sigma_{X} \cdot \sigma_{Y}}\right) \cdot\left(1-\rho^{2}\right)^{-2} \cdot 3 \rho \cdot \sigma_{X}{ }^{3} \cdot \sigma_{Y}+4 \cdot\left(\frac{1}{{\sigma_{Y}}^{2}}\right) \cdot\left(\frac{-\rho}{\sigma_{X} \cdot \sigma_{Y}}\right) \cdot\left(1-\rho^{2}\right)^{-2} \cdot 3 \rho \cdot \sigma_{Y}{ }^{3} \cdot \sigma_{X} \\
+2 .\left(\frac{1}{\sigma_{X}{ }^{2}}\right) \cdot\left(\frac{1}{\sigma_{Y}{ }^{2}}\right)\left(1-\rho^{2}\right)^{-2} \cdot \sigma_{X}{ }^{2} \cdot \sigma_{Y}{ }^{2}\left(1-\rho^{2}\right)+2 \cdot\left(\frac{1}{\sigma_{X}{ }^{2}}\right) \cdot\left(\frac{1}{\sigma_{Y}{ }^{2}}\right)\left(1-\rho^{2}\right)^{-2} \cdot \sigma_{X}{ }^{2} \cdot \sigma_{Y}{ }^{2} \cdot 3 \rho^{2} \\
+12 \cdot\left(\frac{\rho^{2}}{\sigma_{X}{ }^{2} \cdot \sigma_{Y}{ }^{2}}\right)\left(1-\rho^{2}\right)^{-2} \cdot \rho^{2} \cdot \sigma_{X}{ }^{2} \cdot \sigma_{Y}{ }^{2} \\
=\left(1-\rho^{2}\right)^{-2} \cdot\left(8-16 \rho^{2}+8 \rho^{4}\right) \\
=8 \cdot\left(1-\rho^{2}\right)^{-2}\left(1-\rho^{2}\right)^{2} \\
=8
\end{gathered}
$$

Result 5: $E\left(\left(X-\mu_{X}\right)\left(Y-\mu_{Y}\right)\right)=\frac{\lambda}{\lambda_{1}+\lambda_{2}-\lambda}$ 
Result 6: $E\left(\left(X-\mu_{X}\right)^{2}\left(Y-\mu_{Y}\right)^{2}\right)=\frac{1}{\lambda_{1}^{2} \cdot \lambda_{2}{ }^{2}}+\frac{8 \cdot \lambda}{\lambda_{1} \cdot \lambda_{2} \cdot\left(\lambda_{1}+\lambda_{2}-\lambda\right)^{3}}$

Proof:

Note that $E\left(\left(X-\mu_{X}\right)^{2}\left(Y-\mu_{Y}\right)^{2}\right)=E\left(X^{2} \cdot Y^{2}\right)-2 \cdot \mu_{X} \cdot E\left(X \cdot Y^{2}\right)+\mu_{X}{ }^{2} \cdot E\left(Y^{2}\right)$

$$
\begin{aligned}
& -2 \cdot \mu_{Y} \cdot E\left(X^{2} \cdot Y\right)+4 \cdot \mu_{X} \cdot \mu_{Y} \cdot E(X . Y) \\
& -2 \cdot \mu_{X}{ }^{2} \cdot \mu_{Y} \cdot E(Y)+\mu_{Y}{ }^{2} \cdot E\left(X^{2}\right) \\
& -2 \cdot \mu_{X} \cdot \mu_{Y}{ }^{2} \cdot E(X)+\mu_{X}{ }^{2} \cdot \mu_{Y}{ }^{2}
\end{aligned}
$$

One can easily show that for the bivariate exponential density,

(1). $E(X . Y)=\frac{\left(\lambda_{1}+\lambda_{2}\right)}{\lambda_{1} \cdot \lambda_{2} \cdot\left(\lambda_{1}+\lambda_{2}-\lambda\right)}$

(2). $E\left(X^{2} . Y\right)=\frac{6 \cdot\left(\lambda_{1}-\lambda\right)}{\left(\lambda_{1}+\lambda_{2}-\lambda\right)^{4}}+\frac{2 \cdot\left(\lambda_{1}-\lambda\right)}{\lambda_{2} \cdot\left(\lambda_{1}+\lambda_{2}-\lambda\right)^{3}}+\frac{6 \cdot\left(\lambda_{2}-\lambda\right)}{\left(\lambda_{1}+\lambda_{2}-\lambda\right)^{4}}$

$$
+\frac{4 \cdot\left(\lambda_{2}-\lambda\right)}{\lambda_{1} \cdot\left(\lambda_{1}+\lambda_{2}-\lambda\right)^{3}}+\frac{2 \cdot\left(\lambda_{2}-\lambda\right)}{\lambda_{1}^{2} \cdot\left(\lambda_{1}+\lambda_{2}-\lambda\right)^{2}}+\frac{6 \cdot \lambda}{\left(\lambda_{1}+\lambda_{2}-\lambda\right)^{4}}
$$

(3). $E\left(X . Y^{2}\right)=\frac{6 \cdot\left(\lambda_{2}-\lambda\right)}{\left(\lambda_{1}+\lambda_{2}-\lambda\right)^{4}}+\frac{2 \cdot\left(\lambda_{2}-\lambda\right)}{\lambda_{1} \cdot\left(\lambda_{1}+\lambda_{2}-\lambda\right)^{3}}+\frac{6 \cdot\left(\lambda_{1}-\lambda\right)}{\left(\lambda_{1}+\lambda_{2}-\lambda\right)^{4}}$

$$
+\frac{4 \cdot\left(\lambda_{1}-\lambda\right)}{\lambda_{2} \cdot\left(\lambda_{1}+\lambda_{2}-\lambda\right)^{3}}+\frac{2 \cdot\left(\lambda_{1}-\lambda\right)}{\lambda_{2}^{2} \cdot\left(\lambda_{1}+\lambda_{2}-\lambda\right)^{2}}+\frac{6 \cdot \lambda}{\left(\lambda_{1}+\lambda_{2}-\lambda\right)^{4}}
$$

(4). $E\left(X^{2} \cdot Y^{2}\right)=\frac{24}{\left(\lambda_{1}+\lambda_{2}-\lambda\right)^{4}}+\frac{12 \cdot\left(\lambda_{1}-\lambda\right)}{\lambda_{2} \cdot\left(\lambda_{1}+\lambda_{2}-\lambda\right)^{4}}+\frac{12 \cdot\left(\lambda_{2}-\lambda\right)}{\lambda_{1} \cdot\left(\lambda_{1}+\lambda_{2}-\lambda\right)}$

$$
+\frac{4 \cdot\left(\lambda_{1}-\lambda\right)}{\lambda_{2}^{2} \cdot\left(\lambda_{1}+\lambda_{2}-\lambda\right)^{3}}+\frac{4 \cdot\left(\lambda_{2}-\lambda\right)}{\lambda_{1}^{2} \cdot\left(\lambda_{1}+\lambda_{2}-\lambda\right)^{3}}
$$

By substituting these equations in $(A .1)$, we get Result 6 .

Result 7: $E\left(\left(X-\mu_{X}\right)^{3} \cdot\left(Y-\mu_{Y}\right)\right)=\frac{3 \cdot \lambda}{\lambda_{1}^{3} \cdot \lambda_{2} \cdot\left(\lambda_{1}+\lambda_{2}-\lambda\right)}+\frac{6 \cdot \lambda}{\lambda_{1} \cdot \lambda_{2} \cdot\left(\lambda_{1}+\lambda_{2}-\lambda\right)^{3}}$

Proof:

Note that 


$$
\begin{gathered}
E\left(X^{3} . Y\right)=\frac{24}{\left(\lambda_{1}+\lambda_{2}-\lambda\right)^{4}}+\frac{18 \cdot\left(\lambda_{2}-\lambda\right)}{\lambda_{1} \cdot\left(\lambda_{1}+\lambda_{2}-\lambda\right)^{4}}+\frac{12 \cdot\left(\lambda_{2}-\lambda\right)}{\lambda_{1}{ }^{2} \cdot\left(\lambda_{1}+\lambda_{2}-\lambda\right)^{3}}+\frac{6 \cdot\left(\lambda_{1}-\lambda\right)}{\lambda_{2} \cdot\left(\lambda_{1}+\lambda_{2}-\lambda\right)^{4}} \\
+\frac{6 \cdot\left(\lambda_{2}-\lambda\right)}{\lambda_{1}^{3} \cdot\left(\lambda_{1}+\lambda_{2}-\lambda\right)^{2}}
\end{gathered}
$$

By substituting the above equations, we get the result.

Result 8: $E\left(\left(X-\mu_{X}\right) \cdot\left(Y-\mu_{Y}\right)^{3}\right)=\frac{3 \cdot \lambda}{\lambda_{1} \cdot \lambda_{2}{ }^{3} \cdot\left(\lambda_{1}+\lambda_{2}-\lambda\right)}+\frac{6 \cdot \lambda}{\lambda_{1} \cdot \lambda_{2} \cdot\left(\lambda_{1}+\lambda_{2}-\lambda\right)^{3}}$

Proof: Follows by interchanging the variables $X$ and $Y$.

Result 9: Kurtosis, $\kappa=20+\frac{20 \cdot \rho^{2}}{\left(1-\rho^{2}\right)}+\frac{8 \cdot \lambda \cdot\left\{2 \cdot \lambda_{1} \cdot \lambda_{2} \cdot\left(1+2 \cdot \rho^{2}\right)-3 \cdot \rho \cdot\left(\lambda_{1}^{2}+\lambda_{2}^{2}\right)\right\}}{\left(1-\rho^{2}\right)^{2} \cdot\left(\lambda_{1}+\lambda_{2}-\lambda\right)^{3}}$

Proof: Follows from combining all the previous results.

\section{Copyrights}

Copyright for this article is retained by the author(s), with first publication rights granted to the journal.

This is an open-access article distributed under the terms and conditions of the Creative Commons Attribution license (http://creativecommons.org/licenses/by/3.0/). 